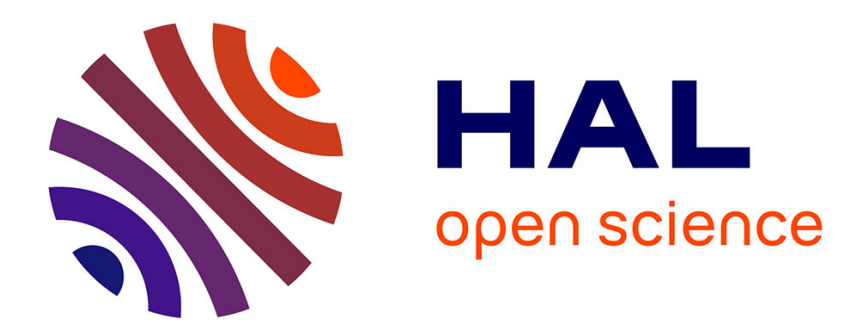

\title{
Le travail d'aimantation des cristaux
}

Pierre Weiss

\section{To cite this version:}

Pierre Weiss. Le travail d'aimantation des cristaux. J. Phys. Theor. Appl., 1904, 3 (1), pp.194-202. 10.1051/jphystap:019040030019401 . jpa-00240865

\section{HAL Id: jpa-00240865 https://hal.science/jpa-00240865}

Submitted on 1 Jan 1904

HAL is a multi-disciplinary open access archive for the deposit and dissemination of scientific research documents, whether they are published or not. The documents may come from teaching and research institutions in France or abroad, or from public or private research centers.
L'archive ouverte pluridisciplinaire HAL, est destinée au dépôt et à la diffusion de documents scientifiques de niveau recherche, publiés ou non, émanant des établissements d'enseignement et de recherche français ou étrangers, des laboratoires publics ou privés. 


\section{LE TRAVAIL D'AIMANTATION DES GRISTAUX;}

Par M. Pierre WEISS.

L'aimantation induite dans un corps cristallisé par un champ de grandeur et de direction données a en général une direction différente de ce champ.

Le problème expérimental qui se pose pour chaque cristal magnétique comprend donc la détermination de trois fonctions de trois variables, les trois composantes de l'aimantation $\mathrm{I}_{x}, \mathrm{I}_{y}, \mathrm{I}_{z}$, en fonction

(1) Ces résultats sont à rapprocher de ceux trouvés par M. et $\mathbf{M}^{\text {me }}$ Huggins, qui ont montré que le spectre de la lumière propre émise spontanément par les sels de radium dans l'air est constitué par les bandes de l'azote.

${ }^{2}$ Ce résultat est en accord avec ceux obtenus par M. Ramsay sur la production de l'hélium par les sels de radium dissous dans l'eau. 
des trois composantes du champ $H_{x}, I_{y}, I_{z}$. Cette étude se simplifie considérablement, et une représentation simple des résultats s'offre immédiatement à l'esprit quand on fait usage de la notion de travail.

On doit, en appliquant les principes de la thermodynamique, tenir compte des échanges de chaleur accompagnant le phénomène étudié $\left({ }^{1}\right)$. Mais deux cas sont particulièrement simples, celui où les phénomènes sont isothermiques et celui où ils sont adiabatiques. Dans le premier, tant qu'ils sont réversibles, le travail dépensé sur le système est égal à l'accroissement de potentiel thermodynamique; dans le deuxième, il est égal à l'accroissement d'énergie. Je fais ici abstraction des phénomènes d'hystérésis; dans l'un et l'autre cas le travailélémentaire d'aimantation sera donc différentielle exacte. Comme, dans la suite, je ne m'appuierai que sur cette proposition, les déductions seront valables pour ces deux cas; mais je conformerai le langage à celui des variations isothermiques.

Je suppose, pour calculer le travail d'aimantation d'un corps, isotrope ou non, que l'on amène successivement de l'infini au point du champ qu'ils occupent tous les éléments de volume $\delta v$ dont le corps se compose, par un mouvement de translation. Le travail des couples agissant sur ces éléments de volume est alors constamment nul, et il ne reste qu'à additionner les travaux des forces. Les composantes rectangulaires de la force agissant sur $\delta v$ sont :

$$
\begin{aligned}
& \delta v \times \mathrm{I}_{x} \frac{\partial \mathrm{H}_{x}}{\partial x}, \\
& \delta v \times \mathrm{I}_{y} \frac{\partial \mathrm{H}_{y}}{\partial y}, \\
& \delta v \times \mathrm{I}_{z} \frac{\partial \mathrm{H}_{z}}{\partial z},
\end{aligned}
$$

et par suite le travail élémentaire recueilli par la main qui effectue la translation est, pour l'une des composantes, $\partial v \times \mathrm{I}_{x} \frac{\partial \mathrm{H}_{x}}{\partial x} d x$. Le travail dépensé total est donc :

$$
\delta \mathrm{T}=-\delta v \int \mathrm{I}_{x} d \mathrm{H}_{x}+\mathrm{I}_{y} d \mathrm{H}_{y}+\mathrm{I}_{z} d \mathrm{H}_{z} .
$$

Ce travail est égal au potentiel thermodynamique provenant de

(') Voir Pellat, J. de Phys., 3e série, VIII, p. 18; 1898. 
l'introduction du corps dans le champ. Il faudrait maintenant sommer tous les $\delta$ T relatifs à tous les éléments de volume; pour simplifier, je ne considérerai que le cas où le champ et l'intensité d'aimantation sont uniformes. Le potentiel thermodynamique rapporté à l'unité de volume est alors :

$$
\mathrm{P}=-\int \mathrm{I}_{x} d \mathrm{H}_{x}+\mathrm{I}_{y} d \mathrm{H}_{y}+\mathrm{I}_{z} d \mathrm{II}_{z}
$$

où chacune des quantités $\mathrm{I}_{x}, \mathrm{I}_{y}, \mathrm{I}_{z}$ est une fonction des trois variables $\mathbf{H}_{x}, \mathbf{H}_{y}, \mathbf{H}_{z}$. Par suite de l'égalité de l'action et de la réaction, on dépensera le même travail, mesuré par $\mathrm{P}$, en supposant la matière à aimanter immobile, et en en approchant l'aimant permanent qui produit le champ de manière à faire passer les composantes de celuici, $\mathrm{H}_{x}, \mathrm{H}_{y}, \mathrm{H}_{z}$, par des valeurs prescrites. $d \mathrm{P}$ est différentielle exacte. $\mathrm{P}$ est négatif pour un corps paramagnétique, positif pour un corps diamagnétique.

La connaissance de $\mathrm{P}$ en fonction de $\mathrm{H}$ renseigne complètement sur les propriétés magnétiques de la substance. Supposons, en effet, que l'on ait réuni par une surface tous les points $\mathbf{I I}_{x}, \mathbf{H}_{y}, \mathbf{H}_{z}$ pour lesquels $P$ a une même valeur, et que l'on trace l'ensemble des surfaces équipotentielles répondant à cette définition. On a, pour une direction quelconque prise comme axe des $x$,

$$
\frac{\partial \mathrm{P}}{\partial \mathrm{H}_{x}}=-\mathrm{I}_{x}
$$

La dérivée de $\mathrm{P}$ dans une direction quelconque est donc égale, au signe près, à la composante de l'aimantation dans cette direction. $\mathrm{Ou}$, en d'autres termes: l'intensité d'aimantation est donnée en direction par la normale à la surface $\mathrm{P}=$ constante, et en grandeur par la dérivée de $\mathrm{P}$, par rapport au champ, prise dans la direction de cette normale.

Le potentiel thermodynamique se décompose en deux termes, en intégrant par parties:

$$
\mathrm{P}=-\left(\mathbf{H}_{x} \mathrm{I}_{x}+\mathrm{H}_{y} \mathrm{I}_{y}+\mathrm{H}_{z} \mathrm{I}_{z}\right)+\int \mathrm{H}_{x} d \mathrm{I}_{x}+\mathbf{H}_{y} d \mathrm{I}_{y}+\mathbf{H}_{z} d \mathrm{I}_{z} .
$$

Pour trouver leur signification, supposons l'aimantation rigide et ayant, avant l'introduction du corps dans le champ, la grandeur et a direction finales, alors le deuxième terme est nul. Le premier 
reste le même, il représente donc le potentiel thermodynamique relatif $\Theta$ du corps aimanté el du champ $\left(^{1}\right)$. Le second sera par définition le potentiel de l'aimantation induite II :

$$
P=\Theta+\text { II. }
$$

$\Theta$ est négatif et II positif pour les corps paramagnétiques.

Considérons maintenant le cas qui se présente dans la pratique expérimentale où le corps exerce sur lui-même un champ démagnétisant uniforme. On pourra choisir les axes de manière que les composantes de ce champ démagnétisant soient données par $\mathrm{N}_{1} \mathrm{I}_{x}$, $\mathrm{N}_{2} \mathrm{I}_{y}, \mathrm{~N}_{3} \mathrm{I}_{3}$, où les trois constantes $\mathrm{N}_{1}, \mathrm{~N}_{2}, \mathrm{~N}_{3}$ sont les trois coefficients démagnétisants principaux. En se reportant à la déduction de la formule (1), on voit que $\mathbf{H}_{r}, \mathbf{H}_{y}, \mathbf{H}_{z}$ représentent les composantes du champ extérieur. Or, en appelant $h_{x}, h_{y}, h_{z}$ les composantes du champ qui règne à l'intérieur de la substance, on a :

$$
\mathrm{H}_{x}=h_{x}+\mathrm{N}_{1} \mathrm{I}_{x} \text {, etc. }
$$

et le potentiel de l'aimantation induite se décompose à son tour en deux termes :

$$
\mathrm{II}=\frac{1}{2}\left(\mathrm{~N}_{1} \mathrm{I}_{x}^{2}-\mathrm{N}_{2} \mathrm{I}_{y}^{2}+\mathrm{N}_{3} \mathrm{I}_{z}^{2}\right)+\int h_{x} d \mathrm{I}_{x}+h_{y} d \mathrm{I}_{y}+h_{z} d \mathrm{I}_{z} .
$$

Le premier est l'énergie mutuelle des différentes parties du corps, et le dernier seul, que nous appellerons potentiel interne d'aimantation, dépend des propriétés spécifiques de la matière. On calculera aisément le premier terme, et on pourra le retrancher de $\Pi$ quand il y aura lieu. Nous supposerons dans la suite le champ démagnétisant négligeable.

La lonction

$$
\Pi=\int \mathrm{I}_{x} d \mathrm{I}_{x}+\mathbf{H}_{y} d \mathrm{I}_{y}+\mathrm{H}_{z} d \mathbf{I}_{z},
$$

dont la connaissance est équivalente à celle de la fonction $\mathrm{P}$, rem-

(1) Quand le champ est produit par des courants, au lieu d'être produit par des aimants, l'énergie relative de la substance aimantée et du courant est nulle : mais le travail des forces est le même et l'énergie reflue, par le mécanisme de l'induction, jusqu'à la source d'électricité. $\Theta$ représenterait donc le potentiel relatif de la substance aimantée et du système producteur du champ en y comprenant la source d'électricité. 
placera avec avantage cette dernière, quand on prendra $\mathrm{I}_{x}, \mathrm{I}_{y}, \mathrm{I}_{z}$ comme variables indépendantes. On démontre en effet, comme pré cédemment, la proposition symétrique de celle énoncée ci-dessus : le champ correspondant à une certaine valeur $\mathrm{I}_{x}, \mathrm{I}_{y}, \mathrm{I}_{z}$ de l'intensité d'aimantation est donné en direction par la normale $\dot{a}$ la surface $\mathrm{II}=$ constante passant par $\mathrm{I}_{x} \mathrm{I}_{y} \mathrm{I}_{z}$, et en grandeur par la dérivée de II prise dans la direction de cette normale.

La connaissance de $\Pi$ en fonction de I renseigne donc complètement sur les propriétés magnétiques de la substance, aussi bien que celle de $\mathrm{P}$ en fonction de $\mathrm{H}$.

Applications aux substances à fonctions magnétisantes linéaires. Supposons qu'une substance prenne sous l'action de la résultante géométrique de deux champs magnétisants une aimantation qui est la résultante géométrique de celle qu'elle prendrait sous l'action de chacun des champs composants.

Il faut et il suffit pour cela que :

$$
\begin{aligned}
& \mathrm{I}_{x}=k_{11} \mathrm{H}_{x}+k_{12} \mathrm{H}_{y}+k_{13} \mathrm{H}_{z} \\
& \mathrm{I}_{y}=k_{21} \mathrm{H}_{x}+k_{22} \mathrm{H}_{y}+k_{23} \mathrm{H}_{z} \\
& \mathrm{I}_{z}=k_{31} \mathrm{H}_{x}+k_{32} \mathrm{H}_{y}+k_{33} \mathrm{H}_{z}
\end{aligned}
$$

où les coefficients $k$ sont constants.

La condition $d \mathrm{P}$ différentielle exacte donne

$$
\frac{\partial \mathrm{I}_{y}}{\partial \mathrm{H}_{z}}=\frac{\partial \mathrm{I}_{z}}{\partial \mathrm{H}_{y}}, \quad \frac{\partial \mathrm{I}_{z}}{\partial \mathrm{H}_{x}}=\frac{\partial \mathrm{I}_{x}}{\partial \overline{\mathrm{H}}_{z}}, \quad \frac{\partial \mathrm{I}_{x}}{\partial \mathrm{H}_{y}}=\frac{\partial \mathrm{I}_{y}}{\partial \mathrm{H}_{x}},
$$

c'est-à-dire :

$$
k_{23}=k_{32}, \quad k_{31}=k_{13}, \quad k_{12}=k_{21},
$$

relations bien connues, déduites pour la première fois de la conservation du travail par lord Kelvin $\left({ }^{1}\right)$, au moyen d'un calcul un peu moins direct, et qui conduisent, pour le potentiel thermodynamique total, ̀̀ :

$$
\mathrm{P}=-\frac{1}{2}\left(k_{11} \mathrm{H}_{x}^{2}+k_{22} \mathrm{H}_{y}^{2}+k_{33} \mathrm{H}_{z}^{2}+2 k_{23} \mathrm{H}_{y} \mathrm{H}_{z}+2 k_{13} \mathrm{H}_{x} \mathrm{H}_{z}+2 k_{12} \mathrm{H}_{x} \mathrm{H}_{y}\right) .
$$

Les surfaces $\mathrm{P}=\mathrm{C}^{\mathrm{te}}$ sont donc des ellipsoïdes homothétiques,

(1) Sir W. Thomsox, Electiostatics and Magnetism, p. 485. 
dont les dimensions linéaires croissent comme $\sqrt{\mathrm{P}}$. Prenons comme axes les axes de l'ellipsoïde, alors $k_{23}=k_{12}=k_{13}=0$.

A chaque direction du champ correspond une aimantation ayant la direction du diamètre conjugué du plan perpendiculaire au champ dans l'ellipsoïde $\mathrm{P}=\mathrm{C}^{\text {te }}$ dont les demi-axes sont $\sqrt{-\frac{2 \mathrm{P}}{k_{11}}}, \sqrt{-\frac{2 \mathrm{P}}{k_{22}}}$, $\sqrt{-\frac{2 P}{k_{33}}}$ La grandeur de l'aimantation pour un champ donné est égale aux rayons vecteurs d'un autre ellipsoïde, ayant pour demiaxes $k_{11} \mathrm{H}, k_{22} \mathrm{H}, k_{33} \mathrm{H}$.

Dans les substances à fonctions magnétisantes linéaires, l'aimantation ne peut donc, à elle seule, mettre en évidence des asymétries supérieures à celle du système orthorhombique.

Si l'on forme $\Theta$ au moyen des équations $(3)$, l'on trouve $\Theta=2 P$, et par suite II $=-\mathrm{P}$.

Le potentiel thermodynamique total est donc égal, au signe près, au potentiel interne d'aimantation et égal en grandeur et en signe à la moitié de l'énergie relative de la substance aimantée et du champ.

Détermination expérimentale du potentiel thermodynamique d'aimantation. - Première méthode. - On peut écrire l'équation (1) sous la forme:

$$
\mathbf{P}=-\int \mathrm{I} \cos (\widehat{\mathrm{I}, d \mathrm{H}}) \cdot d \mathrm{H}
$$

et si l'on fait varier $\mathrm{H}$ en lui laissant une direction constante :

$$
\mathrm{P}=-\int \mathrm{I} \cos \widehat{(\mathrm{I}, \mathrm{H})} d \mathrm{H}
$$

Il suffira donc de relever la composante de l'aimantation parallèle au champ en fonction du champ et d'opérer la quadrature de cette fonction.

Cette mesure, faite pour toutes les directions, dispense donc, en vertu des propriétés du potentiel thermodynamique, de la mesure de l'obliquité de l'aimantation sur le champ.

Deuxième méthode. - Mesurons le couple $\mathrm{C}_{z}$ exercé par le champ sur la substance par rapport à l'axe des $z$. Si nous donnons au champ une petite rotation $d \gamma$ autour de cet axe, le travail dépensé par le 
champ sur la substance sera :

$$
\mathrm{C}_{z} d \gamma=d \mathrm{P}
$$

Introduisons les coordonnées sphériques en appelant $\theta$ l'angle du champ avec le plan des $x y$ et $\gamma$ l'angle de sa projection sur ce plan avec l'axe des $x$, et $\zeta$ et $\varphi$ les quantités analogues pour l'intensité d'aimantation. Ce changement de variable effectué dans $d P$, on a, en tenant compte de ce que $H$ est constant :

$$
\mathrm{C}_{z}=\mathrm{HI} \cos \theta \cos \zeta \sin (\varphi-\gamma) \text {. }
$$

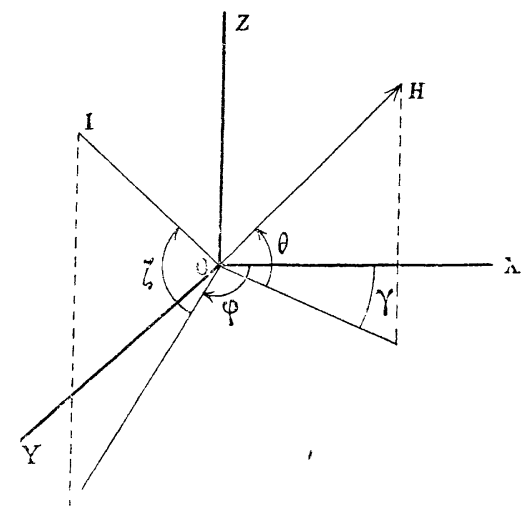

On retrouve ainsi une formule que l'on aurait pu écrire directement d'après la loi d'action d'un champ sur un aimant de moment donné. Je ne la rappelle que pour fixer les idées sur les quantités magnétiques qui interviennent dans $\mathrm{C}_{z}$. Revenant à la formule (̋̃), supposons que, partant d'un point où le potentiel $P_{0}$ est connu pour arriver en un point où il ne l'est pas, on ait fait tourner le champ, supposé constant, d'un angle fini, on aura :

$$
\mathrm{P}-\mathrm{P}_{0}=\int \mathrm{C}_{z} d \gamma
$$

On pourra donc obtenir $\mathrm{P}$ pour toutes les grandeurs et directions du champ combinant la première méthode appliquée à une seule direction avec des mesures de couples, exercés par des champs tournants de grandeur constante. 
L'emploi simultané de ces deux méthodes donnera autant de vérifications que l'on voudra.

Applicalion au cas des substances possédant un plan magnétique comme la pyrrhotine $\left({ }^{1}\right)$. - On aura constamment, pour les substances douées de la propriété de l'aimantation plane, $1 \approx=0$, et la condition $d \mathrm{P}$ différentielle exacte donne :

$$
\frac{\partial \mathrm{l}_{x}}{\partial \mathrm{H}_{z}}=0, \quad \frac{\partial \mathrm{I}_{y}}{\partial \mathrm{H}_{z}}=0 .
$$

La loi cie l'aimantation dans le plan magnétique n'est donc pas influencée par lexistence d'une composante du champ perpendiculaire au plan magnélique.

J'ai découvert cette loi expérimentalement $\left.{ }^{2}\right)$; l'expérience montre qu'elle s'étend même aux phénomènes d'hystérèse sur lesquels la théorie ci-dessus est muette.

$1 l$ devient dès lors superflu d’introduire dans les calculs autre chose que la composante du champ dans le plan magnétique. Soit $\mathrm{H}$

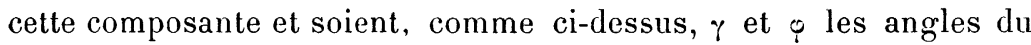
champ et de l'aimantation avec l'axe des $x$; on aura avec les variables II et $\gamma$ :

$$
d P=I I I \sin (\gamma-\varphi) d \gamma+I \cos (\gamma-\varphi) d H,
$$

dont chacun des termes correspond à l'un des modes d'expérimentation indiqués plus haut. La condition $d \mathrm{P}$ différentielle exacte donne

$$
\frac{\partial H I \sin (\gamma-\varphi)}{\partial H}=\frac{\partial I \cos (\gamma-\varphi)}{\partial \gamma}
$$

relation directement vérifiable par l'expérience.

Dans le cas de l'aimantation plane, les surfaces $\mathrm{P}=$ constante se réduisent à des courbes situèes dans le plan magnétique. On peut les considérer comme les courbes de niveau de la surface $\mathrm{P}=f(\mathbf{H}, \gamma)$. La connaissance de cette surface unique, ou celle de la surface $\mathrm{II}=\mathrm{F}(\mathrm{I}, \stackrel{\varphi}{)})$, qui lui est équivalente, épuise complètement la question. L'emploi de la première de ces surfaces et des variables indépendantes $H, \alpha$ pent sembler plus commode par son rattachement direct aux méthodes expérimentales; mais, pour toutes les subs-

(1) Pierre Werss, J. cle Plhys., 3º́nie, VII1, $542 ; 1899$.

$\left({ }^{2}\right)$ Expériences encore inédites. 
tances possédant la propriété de la saturation, la seconde aura l'avantage de donner une représentation complète des propriétés magnétiques ne s'étendant qu'à distance finie.

Exemple de vérification numérique fondée sur l'évaluation du travail: La pyrrhotine possède, dans le plan magnétique, deux directions remarquables rectangulaires pour lesquelles l'aimantation a la direction du champ. Dans l'une d'elles on trouve, pour $\mathbf{H}=3950$ gauss, $P_{1}=-49,0$, pour l'autre $E_{2}=-7,2$ en unités arbitraires. La différence 41,8 est égale, aux erreurs d'expérience près, au travail dépensé en faisant tourner $H$ de l'une à l'autre et qui a été trouvé égal à 42, วั. 